\title{
Prevalência e fatores associados à realização de exames de rastreamento para câncer de próstata em idosos de Juiz de Fora, MG, Brasil
}

\author{
Prevalence and factors associated with conducting screening tests \\ for prostate cancer in the elderly in Juiz de Fora \\ in the state of Minas Gerais, Brazil
}

Lívia Maria Santiago ${ }^{1}$

Laércio Lima Luz ${ }^{2}$

João Francisco Santos da Silva ${ }^{3}$

Inês Echenique Mattos ${ }^{2}$

\footnotetext{
${ }^{1}$ Departamento de

Fonoaudiologia, Faculdade de Medicina, Universidade Federal do Rio de Janeiro. R Rodolpho Paulo Rocco 255/ 9E11, Cidade Universitária. 21.941-913 Rio de Janeiro RJ Brasil.

liviamsantiago@gmail.com ${ }^{2}$ Escola Nacional de Saúde Pública, Fiocruz.

${ }^{3}$ Faculdade de Medicina, Universidade Federal do Mato Grosso do Sul
}

\begin{abstract}
Little is known about screening practices for prostate cancer in elderly Brazilians. The aim of this study was to estimate the prevalence of screening tests for prostate cancer in the elderly in Juiz de Fora in the state of Minas Gerais and to analyze the associated factors. This is a cross-sectional study with 2825 men aged 60 years or older who participated in the vaccination against influenza campaign in 2006. Social and demographic variables and others related to health conditions and the use of health services were analyzed. Poisson regression multivariate analysis was used to analyze associations between the covariates and the dependent variables and the prevalence of the tests was estimated. The median age was 70.0 ( \pm 7.2$)$ years. The prevalence of performing digital rectal examination was $61.0 \%$ and $75.5 \%$ for PSA. The "family history of prostate cancer," "type of health service," "marital status," "regular use of medication" and "education" variables were independent factors associated with performing digital rectal examination. The same variables, with the exception of "marital status" remained in the multiple model for PSA. The study showed that many seniors have adopted the practice of screening and the need to measure and describe this process in view of its possible repercussions on public health.
\end{abstract}

Key words Health of the elderly, Screening, Prostate cancer
Resumo Pouco se conhece sobre as práticas de rastreamento para câncer de próstata em idosos brasileiros. O objetivo deste estudo foi estimar a prevalência de realização de exames de rastreamento para câncer de próstata em idosos de Juiz de Fora (MG) e analisar os fatores associados. Trata-se de estudo seccional com 2825 homens de 60 anos ou mais que participaram da campanha de vacinação contra gripe de 2006. Foram analisadas variáveis sociodemográficas e relativas a condições de saúde e ao uso de serviços de saúde. Utilizou-se a regressão de Poisson na análise multivariada para avaliar associações entre as covariáveis e as variáveis dependentes e estimou-se a prevalência de realização dos exames. A idade média da população foi de 70,0 $( \pm 7,2)$ anos. A prevalência de realização de toque retal foi $61,0 \%$ e a de PSA 75,5\%. As variáveis "história familiar de câncer de próstata", "tipo de serviço de saúde", "status conjugal", "uso de medicação regular" $e$ "escolaridade" foram fatores independentes associados à realização de toque retal. As mesmas variáveis, com exceção do "status conjugal", permaneceram no modelo múltiplo para PSA. O estudo evidencia que muitos idosos têm aderido à prática do rastreamento e a necessidade de dimensionar e qualificar esse processo, tendo em vista suas possíveis repercussões na saúde pública.

Palavras-chave Saúde do idoso, Rastreamento, Câncer de próstata 


\section{Introdução}

O rastreamento de câncer tem como objetivo possibilitar o tratamento de maneira oportuna, com ações menos agressivas, proporcionando maior chance de cura e redução dos custos financeiros reconhecidamente atribuídos à doença em estágios mais avançados ${ }^{1}$.

No rastreamento do câncer de próstata são utilizados dois exames: o toque retal e a dosagem do Antígeno Prostático Específico (PSA). Entretanto, ambos apresentam limitações relacionadas à sensibilidade e especificidade e ao baixo valor preditivo positivo ${ }^{2-4}$. Em função disso, os benefícios e os riscos do rastreamento para este câncer têm sido amplamente debatidos na literatura e não há consenso em relação às diretrizes para sua utilização em nível populacional ${ }^{3-6}$.

De modo geral, é preconizado que os médicos discutam a realização do rastreamento e suas possibilidades diagnósticas e terapêuticas com os pacientes de 50 anos ou mais, para que seja toma$\mathrm{da}$, individualmente, uma decisão informada ${ }^{7,8}$. Na prática, o que tem sido observado em diferentes países é a realização dos exames de rastreamento por uma elevada proporção de indivíduos, com repercussões significativas na saúde pública, como sobrediagnóstico e sobretratamento ${ }^{8-10}$.

No Brasil, em estudo realizado em 2000, foram entrevistados 135 médicos de 51 anos ou mais, professores da Faculdade de Medicina da Universidade Federal de Minas Gerais, sendo que $20,7 \%$ desses relataram nunca haver se submetido ao toque retal ou à dosagem de $\mathrm{PSA}^{11}$. Em um estudo seccional de base populacional realizado em municípios do estado de São Paulo, entre 2001 e 2002, com homens de 50 anos ou mais, verificou-se que 44,4\% dos 992 participantes nunca haviam realizado exame de rastreamento para câncer de próstata ${ }^{12}$. Nesse estudo, a não realização dos exames esteve associada, entre outros fatores, à idade inferior a 70 anos, escolaridade de até 8 anos e renda familiar per capita menor do que 0,5 salário mínimo.

Um incremento substancial da incidência e mortalidade por câncer de próstata é observado com o aumento da idade ${ }^{13}$. Para o ano de 2012, estimou-se que a incidência deste câncer na região sudeste do Brasil variaria entre 67/100.000 e 97/100.000 homens, respectivamente em Minas Gerais e no Rio de Janeiro ${ }^{14}$. Dados do Registro de Câncer de Base Populacional de São Paulo para o período mais recente disponível (2000-2005) indicam que as taxas de incidência de câncer de próstata variaram entre 337,01/100.000 na faixa etária de 60-64 anos e 1.137,56/100.000 na faixa etária de 80-84 anos ${ }^{14}$.

Entretanto, pouco ainda se conhece em relação às práticas de rastreamento de câncer de próstata em homens idosos brasileiros. O presente estudo tem como objetivo estimar a prevalência de realização de exames de rastreamento para câncer de próstata em homens de 60 anos ou mais residentes em um município de médio porte no estado de Minas Gerais e analisar os fatores associados a essa prática.

\section{Material e métodos}

Este é um estudo transversal, desenvolvido com dados do projeto de pesquisa "Envelhecimento populacional e câncer - projeto EPOCA", obtidos em inquérito realizado em Juiz de Fora, Minas Gerais, durante o período de vacinação antiinfluenza dos idosos no ano de 2006. Os métodos de escolha da população e da coleta de dados foram detalhados em publicação anterior ${ }^{15,16}$. Resumidamente, foram convidados a participar da pesquisa indivíduos com idade igual ou superior a 60 anos, de ambos os sexos, que compareceram a um dos 41 postos de vacinação do município. Aqueles que aceitaram participar foram entrevistados com base em um questionário estruturado, que incluía instrumentos padronizados e validados, utilizados em outros estudos nacionais com populações idosas.

No presente estudo, foram incluídos 2.825 homens que responderam a questões relacionadas à realização de exame de toque retal ou PSA, correspondendo a $97,5 \%$ dos indivíduos do sexo masculino que fizeram parte da pesquisa.

As variáveis independentes analisadas foram: sociodemográficas (idade, situação conjugal, escolaridade, trabalho atual); relativas ao uso de serviços de saúde (tipo de serviço de saúde de referência); relativas a condições de saúde (saúde autorreferida, hospitalização recente, morbidade autorreferida, uso de medicação regular e história familiar de câncer de próstata).

As variáveis dependentes consideradas foram a realização de PSA e de toque retal nos últimos cinco anos.

Efetuou-se a análise descritiva das características sociodemográficas e de saúde da população de estudo, segundo a realização dos exames de detecção precoce do câncer de próstata, por meio de distribuição de frequências. Foram verificadas as possíveis associações entre a realização dos exames e as variáveis independentes, por meio de 
estimativas das razões de prevalência (RP) e seus respectivos intervalos de confiança (IC) de 95\%.

Para explorar as associações entre a realização de toque retal e de PSA e as variáveis independentes utilizou-se a análise múltipla de regressão de Poisson. Considerou-se nível de significância estatística de 0,05 para que as variáveis fossem mantidas nos modelos finais.

As análises estatísticas foram efetuadas utilizando-se os pacotes SPSS 17.0 (Statistical Package Social Science) e o STATA 10 for Windows.

\section{Resultados}

A média de idade dos 2.825 idosos do estudo foi de 70,0 ( $\pm 7,2)$ anos e a mediana de 69,0 anos. Considerando os cinco anos anteriores à data de entrevista, a prevalência de realização de toque retal foi $61,0 \%$ e a de PSA correspondeu a 75,5\%.

A prevalência de realização de toque retal foi similar nas três faixas etárias. Em relação ao status conjugal, os idosos casados ou com companheira tinham maior probabilidade de ter realizado esse exame em comparação com os solteiros. A maior escolaridade, a utilização de serviço de saúde privado, o relato de pelo menos uma morbidade, o uso de medicação regular e a história familiar de câncer de próstata também mostraram associações positivas e estatisticamente significativas com a realização de toque retal (Tabela 1).

Em relação ao PSA, observou-se que os indivíduos mais jovens (60-69 e 70-79 anos) tinham maior probabilidade de ter realizado o exame, quando comparados aos de 80 anos ou mais. $\mathrm{O}$

Tabela 1. Razões de prevalência brutas e ajustadas de realização de toque retal por variáveis sociodemográficas e de saúde, Juiz de Fora (MG), Brasil, 2006. ( $\mathrm{N}=2825)$.

\begin{tabular}{|c|c|c|c|}
\hline Variáveis & $\mathrm{N}$ total & Prevalência de toque retal (\%) & RP bruta (IC 95\%) \\
\hline \multicolumn{4}{|l|}{ Faixa etária } \\
\hline 60 a 69 anos & 1486 & $898(60,4)$ & 1 \\
\hline 70 a 79 anos & 1009 & $630(62,4)$ & $1,05(0,95-1,16)$ \\
\hline 80 anos ou mais & 330 & $196(59,4)$ & $1,02(0,92-1,12)$ \\
\hline \multicolumn{4}{|l|}{ Status conjugal } \\
\hline Solteiro/divorciado & 376 & $190(50,5)$ & 1 \\
\hline Viúvo & 367 & $207(56,4)$ & $1,12(0,98-1,28)$ \\
\hline Casado/companheira & 2073 & $1321(63,7)$ & $1,26(1,14-1,40)$ \\
\hline \multicolumn{4}{|l|}{ Escolaridade } \\
\hline$\leq 4$ anos & 2066 & $1186(57,4)$ & 1 \\
\hline$>4$ anos & 756 & $536(70,9)$ & $1,24(1,16-1,31)$ \\
\hline \multicolumn{4}{|l|}{ Trabalho atual } \\
\hline Não & 2110 & $1289(61,1)$ & 1 \\
\hline Sim & 700 & $427(61,0)$ & $1,00(0,93-1,07)$ \\
\hline \multicolumn{4}{|c|}{ Tipo de serviço de saúde utilizado } \\
\hline Público (SUS) & 1850 & $1030(55,7)$ & 1 \\
\hline Privado & 970 & $693(71,4)$ & $1,28(1,21-1,36)$ \\
\hline \multicolumn{4}{|l|}{ Saúde autorreferida } \\
\hline Razoável/ Ruim & 1044 & $631(60,4)$ & 1 \\
\hline Excelente/Boa & 1760 & $1083(61,5)$ & $1,02(0,96-1,08)$ \\
\hline \multicolumn{4}{|l|}{ Hospitalização recente } \\
\hline Não & 2269 & $1363(60,1)$ & 1 \\
\hline Sim & 542 & $349(64,4)$ & $1,07(1,00-1,15)$ \\
\hline \multicolumn{4}{|l|}{ Morbidades autorreferidas } \\
\hline Não & 798 & $460(57,6)$ & 1 \\
\hline Sim & 2027 & $1264(62,3)$ & $1,08(1,01-1,16)$ \\
\hline \multicolumn{4}{|l|}{ Uso de medicação regular } \\
\hline Não & 813 & $441(54,2)$ & 1 \\
\hline Sim & 1926 & $1224(63,6)$ & $1,17(1,09-1,26)$ \\
\hline \multicolumn{4}{|c|}{ História familiar de câncer de próstata } \\
\hline Não & 2549 & $1526(59,9)$ & 1 \\
\hline Sim & 239 & $174(72,8)$ & $1,22(1,12-1,32)$ \\
\hline
\end{tabular}


status conjugal casado/com companheira, a escolaridade acima de 4 anos, a utilização de serviço de saúde privado, o relato de pelo menos uma morbidade e a história familiar de câncer apresentaram associação positiva com a realização do exame (Tabela 2).
$\mathrm{Na}$ análise multivariada, as variáveis "história familiar de câncer de próstata", "tipo de serviço de saúde", "status conjugal", "uso de medicação regular" e "escolaridade" foram fatores independentes associados à realização de toque retal (Tabela 3). As mesmas variáveis, com exceção do "status

Tabela 2. Razões de prevalência brutas e ajustadas de realização de PSA por variáveis sociodemográficas e de saúde, Juiz de Fora (MG), Brasil, 2006. ( $\mathrm{N}=2825)$.

\begin{tabular}{|c|c|c|c|}
\hline Variáveis & $\mathrm{N}$ total & Prevalência de PSA (\%) & RP bruta (IC 95\%) \\
\hline \multicolumn{4}{|l|}{ Faixa etária } \\
\hline 60 a 69 anos & 330 & $228(69,1)$ & 1 \\
\hline 70 a 79 anos & 1009 & $775(76,8)$ & $1,11(1,03-1,20)$ \\
\hline 80 anos ou mais & 1486 & $1131(76,1)$ & $1,10(1,02-1,19)$ \\
\hline \multicolumn{4}{|l|}{ Status conjugal } \\
\hline Solteiro/divorciado & 376 & $256(68,1)$ & 1 \\
\hline Viúvo & 367 & $269(73,3)$ & $1,08(0,98-1,18)$ \\
\hline Casado/companheira & 2073 & $1601(77,2)$ & $1,13(1,05-1,22)$ \\
\hline \multicolumn{4}{|l|}{ Escolaridade } \\
\hline$\leq 4$ anos & 2066 & $1478(71,5)$ & 1 \\
\hline$>4$ anos & 756 & $653(86,4)$ & $1,21(1,16-1,26)$ \\
\hline \multicolumn{4}{|l|}{ Trabalho atual } \\
\hline Não & 2110 & $1577(74,7)$ & 1 \\
\hline Sim & 700 & $545(77,9)$ & $1,04(0,99-1,09)$ \\
\hline \multicolumn{4}{|c|}{ Tipo de serviço de saúde utilizado } \\
\hline Público (SUS) & 970 & $835(86,1)$ & 1 \\
\hline Privado & 1850 & $1298(70,2)$ & $1,23(1,18-1,28)$ \\
\hline \multicolumn{4}{|l|}{ Saúde autorreferida } \\
\hline Razoável/ Ruim & 1760 & $1325(75,3)$ & 1 \\
\hline Excelente/Boa & 1044 & $797(76,3)$ & $1,01(0,97-1,06)$ \\
\hline \multicolumn{4}{|l|}{ Hospitalização recente } \\
\hline Não & 2269 & $1692(74,6)$ & 1 \\
\hline Sim & 542 & $429(79,2)$ & $1,02(0,97-1,08)$ \\
\hline \multicolumn{4}{|l|}{ Morbidades autorreferidas } \\
\hline Não & 798 & $568(71,2)$ & 1 \\
\hline Sim & 2027 & $1566(77,3)$ & $1,09(1,03-1,14)$ \\
\hline \multicolumn{4}{|l|}{ Uso de medicação regular } \\
\hline Não & 813 & $553(68,0)$ & 1 \\
\hline Sim & 1926 & $1512(78,5)$ & $1,15(1,09-1,22)$ \\
\hline \multicolumn{4}{|c|}{ História familiar de câncer de próstata } \\
\hline Não & 2549 & $1900(74,5)$ & 1 \\
\hline Sim & 239 & $206(86,2)$ & $1,16(1,09-1,22)$ \\
\hline
\end{tabular}

Tabela 3. Modelo de regressão múltipla de Poisson para a realização de toque retal, Juiz de Fora (MG), Brasil, 2006. $(\mathrm{N}=2825)$.

\section{Variável}

História familiar de câncer de próstata (Sim)

Tipo de serviço de saúde que utiliza (Privado)

Status conjugal (Casado/com companheira/viúvo)

Uso de medicação regular (Sim)

Escolaridade ( $>4$ anos)

\section{Razões de Prevalência ajustadas* (IC 95\%)}

$1,20(1,11-1,30)$
$1,17(1,08-1,26)$
$1,17(1,06-1,22)$
$1,16(1,08-1,24)$
$1,14(1,07-1,22)$

*Ajustadas por todas as variáveis no modelo. 
conjugal", permaneceram no modelo múltiplo da análise de regressão para o PSA (Tabela 4).

\section{Discussão}

Atualmente, não se dispõe de evidências suficientes que suportem a recomendação de rastreamento populacional de rotina para câncer de próstata, através do toque retal ou da dosagem de Antígeno Prostático Específico (PSA) ${ }^{17,18}$. No Brasil, o Instituto Nacional do Câncer recomenda que homens de 50 a 70 anos, ao serem atendidos nos serviços de saúde, recebam informações sobre as limitações, os riscos e os benefícios da detecção precoce deste tipo de câncer, para que possam optar pela realização ou não desses exames ${ }^{19}$.

O substantivo impacto do câncer de próstata na população e a ausência de consenso quanto ao rastreamento populacional tem ocasionado inconsistências quanto a essa prática. Assim, em diferentes países tem sido observado que uma elevada proporção de indivíduos realiza esses exames, com repercussões significativas na saúde pública, como sobrediagnóstico e sobretratamento ${ }^{9,10}$. Entretanto, mesmo sendo o câncer de próstata uma doença reconhecidamente associada ao envelhecimento, são poucos os estudos que consideraram as particularidades das populações idosas em relação ao rastreamento oportunístico deste tumor.

Neste estudo observou-se alta prevalência de realização de toque retal e de PSA nos últimos cinco anos entre homens idosos. Entretanto, como os indivíduos entrevistados estavam participando de uma campanha de saúde, é possível que apresentassem particularidades, como um nível diferenciado de cuidado, em comparação com o conjunto da população da mesma faixa etária e sexo.

A prevalência de realização de exames de rastreamento para câncer de próstata tem sido investigada em outros estudos realizados no Bra- sil, porém em populações com características distintas, dificultando a comparação entre os resultados. Em estudo realizado em municípios do estado de São Paulo, foram incluídos homens a partir de 50 anos $^{12}$. Em Minas Gerais, além de serem também estudados homens a partir de 50 anos, todos eram médicos que lecionavam em uma faculdade de Medicina ${ }^{11}$. Nos dois estudos citados, a prevalência de realização de exames de rastreamento para câncer de próstata foi menor que a observada entre os idosos de Juiz de Fora.

Em estudo realizado nos Estados Unidos, foram avaliadas as características associadas à realização de PSA nos 12 meses que antecederam a entrevista em homens de 40 anos ou mais ${ }^{1}$. Nesse estudo, a prevalência de realização de PSA foi estimada para diferentes faixas etárias, sendo observado que aproximadamente $67 \%$ dos participantes de 60 ou mais anos haviam realizado o exame no período de referência. Em outro estudo norte-americano, a prevalência de realização de rastreamento para câncer de próstata foi avaliada com base em dados censitários referentes ao ano $2000^{20}$. Entre indivíduos com idade igual ou superior a 65 anos e possuidores de seguro de saúde, a prevalência estimada de realização de PSA correspondeu a $61,9 \%$ e de toque retal a $57,7 \%$, considerando exames realizados nos últimos 12 meses $^{20}$.

A história familiar de câncer de próstata é um fator de risco reconhecido para a doença e é recomendado que, nesta situação, o rastreamento seja iniciado em torno dos $45 \operatorname{anos}^{8,21,22}$. Tem sido apontado na literatura que indivíduos com história familiar de câncer de próstata se percebem como grupo de maior risco e por isso buscam informações sobre a doença, ou as recebem com maior frequência de seus médicos ou familiares, sendo orientados a realizar exames de rastreamento ${ }^{21,23}$.

Em estudo de Bloom et al. ${ }^{21}$, realizado com 205 afro-americanos, de 40 a 74 anos, a associação entre história familiar de câncer de próstata e

Tabela 4. Modelo de regressão múltipla de Poisson para realização de PSA, Juiz de Fora (MG), Brasil, 2006. $(\mathrm{N}=2825)$.

\begin{tabular}{lc}
\hline \multicolumn{1}{c}{ Variável } & Razões de Prevalência ajustadas ${ }^{*}$ (IC 95\%) \\
\hline Tipo de serviço de saúde que utiliza (Privado) & $1,15(1,11-1,20)$ \\
Uso de medicação regular (Sim) & $1,15(1,09-1,21)$ \\
Escolaridade (> 4 anos) & $1,14(1,09-1,19)$ \\
História familiar de câncer de próstata (Sim) & $1,13(1,07-1,19)$
\end{tabular}

*Ajustadas por todas as variáveis no modelo. 
realização de PSA se manteve estatisticamente significativa, após ajustamento por diversas variáveis sociodemográficas, de saúde e de acesso aos serviços (OR 3,03 IC95\% 1,13-8,10). Em outro estudo, que investigou fatores psicossociais associados à realização de toque retal e de PSA, com 2477 norte-americanos caucasianos, entre 40 a 79 anos, essa associação também manteve significância estatística após ajustamento por idade, nível educacional e preocupação sobre o câncer (OR=1,4 IC95\% 1,0-1,9) ${ }^{24}$. No presente estudo, à semelhança do que foi observado na literatura, a história familiar de câncer de próstata se manteve associada à realização de PSA, mesmo após o ajustamento pelas demais covariáveis. Os dois outros estudos brasileiros identificados, não incluíram essa variável nas suas análises ${ }^{11,12}$.

Observou-se associação entre prevalência de realização dos exames de rastreamento e estado conjugal "casado/com companheira". Entretanto, na análise multivariada, essa associação manteve significância estatística somente para a realização de toque retal. Em estudo realizado em um condado de Minnesota, Estados Unidos, a presença de companheira foi um dos determinantes da realização dos exames de rastreamento (OR 1,2 IC95\% 0,9-1,7), porém perdeu a significância estatística no modelo múltiplo ${ }^{24}$. No estudo norte-americano com homens de 40 anos ou mais, anteriormente citado, foi identificada associação entre realização de PSA e status conjugal casado, ajustada por idade (OR 2,1 IC 1,3-3,5) ${ }^{1}$. A situação conjugal não apresentou associação estatisticamente significativa com a realização de rastreamento no estudo de São Paulo ${ }^{12}$.

Em nosso país, ao avaliar acesso e utilização de serviços de saúde na perspectiva do gênero, se observa a menor presença masculina nos serviços de atenção primária, assim como um diferencial na motivação para a busca dos serviços, com maior procura por exames preventivos e de rotina entre as mulheres e busca por motivo de doença pelos homens ${ }^{25,26}$. As práticas de autocuidado e prevenção da saúde são mais difundidas no universo feminino e é possível que os homens que têm esposa ou companheira sejam influenciados por suas parceiras nas decisões de procurar orientação médica e de realizar exames de rastreamento.

Neste estudo, o tipo de serviço de saúde de referência utilizado foi um fator determinante para a realização de toque retal e de PSA. Os usuários de serviços privados tiveram probabilidade $18 \%$ maior de ter realizado toque retal e $21 \%$ maior de ter realizado PSA, em comparação aos usuários do serviço público. Amorim et al. $.^{12} \mathrm{ob}-$ servaram em seu estudo que o sistema complementar de saúde foi o principal responsável pela oferta de exames de rastreamento para câncer de próstata (59\%). Na investigação de Merril ${ }^{1}$, realizada nos Estados Unidos, ter seguro médico foi um fator fortemente associado à realização do PSA (OR=3,0 IC95\% 1,0-8,9). Da mesma forma, em estudo realizado no Colorado/EUA, com 4.771 homens, entre 50 e 74 anos verificou-se que usuários de rede privada de saúde apresentavam maior probabilidade de realizar toque retal (OR 1,76 IC95\% 1,08-2,89) e PSA (OR 1,84 IC95\% 1,13-2,99), após ajustamento por variáveis sociodemográficas e de saúde ${ }^{27}$. A associação entre maior escolaridade e a realização de toque retal observada neste estudo, foi verificada por outros autores ${ }^{1,9,12,24}$. O nível educacional mais alto supõe maior conhecimento acerca das doenças, entre elas o câncer de próstata, o que poderia levar à maior probabilidade de realização dos exames, bem como de manter outras práticas relacionadas ao autocuidado. No caso específico do Brasil, essa situação pode estar relacionada, em parte, a não recomendação de rastreamento na rede pública ${ }^{19}$.

É possível que a variável "uso regular de medicação", associada à realização dos dois exames em questão em nosso estudo, se constitua em uma medida indireta da utilização de serviços de saúde, principalmente de consulta médica, já que pressupõe uma prescrição por parte deste profissional, com um mínimo de regularidade. As visitas ao médico aumentariam a probabilidade do indivíduo receber indicação para realização desses exames. No estudo de São Paulo, foram observadas associações entre presença de comorbidades e realização de rastreamento, sendo que a variável Diabetes mellitus permaneceu com significância estatística no modelo final ${ }^{12}$. Ao comentar esses achados, os autores sugerem que a maior frequência de visitas aos serviços de saúde por parte desses indivíduos os torne mais expostos à oferta de exames.

Diversos estudos realizados em outros países e não específicos para a população idosa apontam a maior realização dos exames de rastreamento para os tumores de próstata com o aumento da idade ${ }^{1,9,24,27}$. Em São Paulo, também em estudo não específico para a população idosa, essa associação foi igualmente observada ${ }^{12}$. Não é possível fazer uma comparação direta com o presente estudo que incluiu somente indivíduos de 60 ou mais anos de idade. Entretanto, verifica-se que a idade mais elevada, em função das 
condições crônicas e/ou agudas, que comumente afetam os idosos, leva à maior necessidade de acompanhamento médico e de utilização de serviços de saúde, o que poderia se constituir em maior probabilidade de receber indicação desses exames. A maior incidência de câncer de próstata nas faixas etárias mais elevadas poderia motivar a maior frequência de recomendação de exames de rastreamento nesse grupo populacional.

O objetivo deste estudo foi estimar a prevalência da utilização dos exames de toque retal e PSA em homens idosos. Identificou-se uma grande parcela de indivíduos que haviam realizado os exames, embora não haja indicação de rastreamento populacional para câncer de próstata, configurando uma importante demanda individual. Outra constatação interessante foi o fato de que ser usuário do Sistema Único de Saúde não foi um fator de impedimento para o acesso e a realização do rastreamento. A prevalência de realização de PSA foi inclusive maior entre os usuários da rede pública.

Este estudo apresenta limitações, entre elas o desenho transversal que limita a interpretação das associações observadas em termos de causalidade. Outra questão diz respeito ao fato das informações sobre realização dos exames de rastreamento ter sido autorreferida, não sendo possível descartar a introdução de viés de informação. Os indivíduos entrevistados constituem um grupo diferenciado, que estava participando de uma ação preventiva de saúde e, nesse sentido, é possível que a prevalência observada de realização dos exames de rastreamento esteja superestimada em relação ao conjunto da população idosa masculina da cidade.

Apesar das diferenças metodológicas na constituição das populações de estudo, quanto à faixa etária e outras características sociodemográficas, as associações aqui observadas, em sua maioria, são corroboradas por outras investigações.

É importante a realização de estudos de base populacional, para que se possa melhor dimensionar e qualificar o processo de rastreamento de câncer de próstata em idosos brasileiros e suas possíveis repercussões na saúde pública.

\section{Colaboradores}

LM Santiago e IE Mattos trabalharam na concepção do estudo, definição de seu desenho, pesquisa bibliográfica, análise de dados, redação e revisão do artigo. LL Luz e JFS Silva participaram da pesquisa bibliográfica, redação e revisão do artigo. 


\section{Referências}

1. Merrill RM. Demographics and health-related factors of men receiving Prostate-specific Antigen screening in Utah. Prev Medicine 2001; 33(6):646-652.

2. Thompson IM, Ankerst DP, Chi C, Scott LM, Goodman PJ, Crowley JJ, Parnes HL, Coltman CA Junior. Operating Characteristics of Prostate-Specific Antigen in Men With an Initial PSA Level of $3.0 \mathrm{ng} /$ $\mathrm{mL}$ or Lower. JAMA 2005; 294(1):66-70.

3. Crawford ED, Abrahamsson P. PSA-based screening for prostate cancer: how does it compare with other cancer screening tests? Europ Urol 2008; 54(2):262-273.

4. Bryant RJ, Hamdy FC. Screening for prostate cancer: an update. Eur Urol 2008; 53(1):37-44.

5. Schroder FH, Hugosson J, Roobol MJ, Tammela TLJ, Ciatto S, Nelen V, V, Kwiatkowski M, Lujan M, Lilja H, Zappa M, Denis LJ, Recker F, Berenguer A, Määttänen L, Bangma $\mathrm{CH}$, Aus G, Villers A, Rebillard X, van der Kwast T, Blijenberg BG, Moss SM, de Koning HJ, Auvinen A, ERSPC Investigators. Screening and prostate-cancer mortality in a randomized European study. $N$ Engl J Med 2009; 360(13):1320-1328.

6. Greene KL, Abertsen PC, Babaian RJ, Carter HB, Gann PH, Han M, Kuban DA, Sartor AO, Stanford JL, Zietman A, Carroll P. Prostate Specific Antigen best practice statement: 2009 update. J Urology 2009; 182(5):2232-2241.

7. Abrahamsson P, Artibani W, Chapple CR, Wirth M. European Association of Urology Position Statement on Screening for Prostate Cancer. Eur Urol 2009; 56(2):270-271.

8. Smith RA, Cokkinides V, Brooks D, Saslow D, Shah $\mathrm{M}$, Brawley OW. Cancer screening in the United States, 2011. A Review of Current American Cancer Society Guidelines and Issues in Cancer Screening. CA Cancer J Clin 2011; 61(1):18-30

9. Rutten LJF, Meissner HI, Breen N, Vernon SW, Rimer BK. Factors associated with men's use of prostate-specific antigen screening: evidence from Health Information National Trends Survey. Prev $\mathrm{Me}$ dicine 2005; 40(4):461-468.

10. Boyle P. Prostate cancer: current evidence weights against population screening. CA Cancer J Clin 2009; 59(4):220-224.

11. Miranda PSC, Cortes MCJW, Martins ME, Chaves PC, Santarosa RC. Práticas de diagnóstico precoce de câncer de próstata entre professores da faculdade de medicina -UFMG. Rev Assoc Med Bras 2004; 50(3):272-275.

12. Amorim VMSL, Barros MBA, César CLG, Goldbaum M, Carandina L, Alves MCGP. Fatores associados à realização dos exames de rastreamento para o câncer de próstata: um estudo de base populacional. Cad Saude Publica 2011; 27(2):347-356.

13. Swaminathan V, Audisio R. Cancer in older patients: an analysis of elderly oncology. E-cancer medical science 2012; 6:243.

14. Instituto Nacional do Câncer. Estimativa 2012: Incidência de câncer no Brasil. Rio de Janeiro (Brasil): INCA; 2012. [acessado 2013 set 22]. Disponível em: http://wwwl.inca.gov.br/estimativa/2012/mapa. asp?ID=13
15. Novaes CO, Mattos IE. Prevalência e fatores associados a não utilização de mamografia em mulheres idosas. Cad Saude Publica 2009; 25(Supl. 2):s310s320.

16. Santiago LM, Novaes CO, Mattos IE. Factors Associated with Self-Rated Health among Older Men in a Medium-Size City in Brazil. J Men's Health 2010; 7(1):55-63

17. Lin K, Lipsitz R, Miller T, Janakiraman S, US Preventive Services Task Force. Benefits and harms of prostate-specific antigen screening for prostate cancer: an evidence update for US Preventive Services Task Force. Ann Intern Med 2008; 149(3):192-199.

18. Wever EM, Draisma G, Heijnsdijk EAM, Roobol MJ, Boer R, Otto SJ, de Koning HJ. Prostate-Specific Antigen Screening in United States vs in the European Randomized Study of screening for prostate cancer- Rotterdam. JNCI 2010; 102(5):352-355.

19. Instituto Nacional de Câncer (INCA). Programa Nacional de Controle do Câncer da Próstata: documento de consenso. Rio de Janeiro (Brasil): INCA; 2002.

20. Smith RA. Cancer screening in the USA. J Med Screen 2006; 13(Supl. 1):S48-S53.

21. Bloom JR, Stewart SL, Oakley-Girvans IN, Banks PJ, Chang S. Family history, perceived risk, and prostate cancer screening among African American men. Cancer Epidemiol Biomarkers Prev 2006; 15(11): 2167-2173.

22. Park KK, Lee SH, Choi YD, Chung B. Optimal baseline Prostate-Specific Antigen level to distinguish risk of prostate cancer in healthy men between 40 and 69 years of age. J Korean Med Sci 2012; 27(1):40-45.

23. Linder SK, Hawley ST, Cooper CP, Scholl LE, Jibaja-Weiss M, Volk RJ. Primary care physicians' reported use of pre-screening discussions for prostate cancer screening: a cross-sectional survey. Fam Pract 2009; 10:19.

24. Wallner LP, Sarma AV, Lieber MM, Sauver JLSt, Jacobson DJ, McGree ME, Gowan ME, Jacobsen SJ. Psychosocial factors associated with an increased frequency of prostate cancer screening in men ages 40-79: the Olmsted County Study. Cancer Epidemiol Biomarkers Prev 2008; 17(12):3588-3592.

25. Pinheiro RS, Viacava F, Travassos C, Brito AS. Gênero, morbidade, acesso e utilização de serviços de saúde no Brasil. Cienc Saude Colet 2002; 7(4):687-707.

26. Figueiredo W. Assistência à saúde dos homens: um desafio para os serviços de atenção primária. Cienc Saude Colet 2005; 10(1):105-109.

27. Moran WP, Cohen SJ, Preisser JS, Wofford JL, Shelton BJ, McClatchey MW. Factors influencing use of the Prostate-Specific Antigen Screening Test in Primary Care. Am J Manag Care 2000; 6(3):315-324.

Artigo apresentado em 09/06/2012

Aprovado em 25/07/2012

Versão final apresentada em 09/08/2012 